\title{
A Comparative Study of Methods for Modelling the Structural Stiffness of Generator Components
}

\author{
P. Jaen Sola*, A.S. McDonald ${ }^{\dagger}$ \\ Wind Energy Systems Doctoral Training Centre, Department of Electronic and Electrical Engineering, University of \\ Strathclyde, Glasgow, UK, ${ }^{\star}$ pablo.jaen-sola@strath.ac.uk, ${ }^{\dagger}$ alasdair.mcdonald@strath.ac.uk
}

Keywords: Structural stiffness, modelling, direct-drive, radial-flux permanent magnet generator.

\begin{abstract}
Direct-drive generators are low speed electrical machines requiring robust and large supporting structures designed to resist the significant loads present during assembly and operation. Generator structures have to be stiff, especially in the radial direction for radial-flux machines. This paper presents three different structural modelling approaches: finite element, analytical and hybrid (a combination of the results obtained from dimensional studies and finite element analyses). These are used along with models of electromagnetic active material, to parametrically calculate the minimum structural stiffness and mass of the components forming the machine.
\end{abstract}

\section{Introduction}

With a configuration that removes the gearbox from the wind turbine powertrain, direct-drive generators can achieve higher energy yields as well as having the potential for higher availability. However the use of this type of machine introduces new challenges that need to be addressed. Directdrive generators operate at a low speed and so the machines have very high torque ratings. Due to this large machine diameters are necessary and significant radial and tangential forces are present. Taking into consideration that a number of loads of large magnitude act on the rotor and stator, only a very stiff and generally heavy machine is able to deal with the forces and keep the air-gap open and stable. In radial-flux machines it is the radially orientated force that tends to close the air-gap; this force comes from the normal component of Maxwell stress and is proportional to the square of the air-gap flux density.

Different rotor and stator structures have been studied in other papers [1], with the main aim of finding an arrangement that can meet all the requirements at the lowest cost. Bearing this in mind, design engineers have tried to minimize the structural mass of the generator while providing sufficient stiffness. For large direct-drive generators, the structural material dominates the total generator mass [2]. In [3], JaenSola and McDonald presented an electromagnetic model that can be used to estimate the magnetic stiffness and hence the generator structural stiffness. The authors coupled the said electromagnetic model with a parametric structural model, which was developed using finite element techniques. They also came up with a hybrid method that utilises the results obtained from the simulation analyses and the data retrieved from dimensional studies to predict the necessary stiffness of the generator components. This paper carries on that work and shows three distinct approaches that can be applied to calculate the required generator structural stiffness with a minimized mass.

\section{Methodology}

Stiffness is defined as the ratio of force to deflection; stiffer components require a larger force to deflect by the same amount as a more compliant component. In the case of a machine air-gap, as the clearance reduces, the closing force increases.

In this investigation, a radial-flux permanent magnet generator formed by four main elements has been assumed. In terms of stiffness they are as follows: the bearing, $k_{\mathrm{b}}$, the structure of the rotor, $k_{\mathrm{s}, \mathrm{r}}$, the magnetic air-gap stiffness, $k_{\mathrm{M}}$, and the structure of the stator, $k_{\mathrm{s}, \mathrm{s}}$. Combining the bearing and the rotor structure in series gives,

$$
k_{\mathrm{eq}, \mathrm{r}}=\frac{k_{\mathrm{s}, \mathrm{r}} k_{\mathrm{b}}}{k_{\mathrm{s}, \mathrm{r}}+k_{\mathrm{b}}}
$$

where $k_{\text {eq,r }}$ is the equivalent rotor stiffness. To maintain the integrity of the electrical machine, the air-gap must remain open and stable. Considering the radial forces acting on the generator components, an expression defining the required structural stiffness in terms of magnetic air-gap stiffness can be derived,

$$
\frac{k_{\mathrm{eq}, \mathrm{r}} k_{\mathrm{s}, \mathrm{s}}}{k_{\mathrm{eq}, \mathrm{r}}+k_{\mathrm{s}, \mathrm{s}}} \geq k_{\mathrm{M}}
$$


In order to satisfy equation (2) with the minimum mass, it is necessary to find a way of evaluating the structural stiffness and mass of the rotor and stator structures.

There exist different procedures to calculate the structural stiffness needed by an electrical machine. A hierarchy can be defined considering the advantages and drawbacks of each one. Among the three techniques described here, the most sophisticated (and one could argue, more accurate) is the Finite Element method due to its ability to capture geometric, loading and material features. However, this approach is computationally expensive and time consuming and is more suited to final design evaluation, rather than early optimisation.

The analytical methods presented in this paper are capable of producing accurate results for Mode 0 (uniform radial loading) and Mode 1 (sinusoidal radial loading due to eccentricity) deflection of sub-structures, such as arms, in a much faster way. In order to calculate stiffness of the structure it is often necessary to combine the stiffness of different sub-structures in series. Generally these analytical techniques are accurate when the geometry is simple and the loading is Mode 0 .

The hybrid procedure combines a limited number of FE results and fits functions to the results. This lets a designer (or optimisation procedure) to evaluate the design space in a continuous albeit approximate fashion. It is more suited to sub-structures as additional independent variable make the function fitting much more challenging.

\subsection{Case Study Wind Turbine Generator and its Structures}

Figure 1 shows two alternative rotor structures for a $3 \mathrm{MW}$ electrical machine of $4 \mathrm{~m}$ diameter and $1.2 \mathrm{~m}$ of axial length that have been used as a case study in this paper. The stiffness of this machine, made up of steel, can be altered by changing its dimensions. Real generator structures can be sophisticated, whereas Figure 1 shows two simplified structures. In the disc structure case, the rotor cylinder thickness $t_{\mathrm{c}}$ and rotor disc thickness $t_{\mathrm{d}}$ can be varied. For the rotor with an armed structure, it is the cylinder thickness and the wall thickness of the arms, $t_{\mathrm{arm}}$ that are varied. Similar models have been developed for the outer stator structures. Depending on the mode of deflection, the air-gap flux density ranges from 0.92 to $1.02 \mathrm{~T}$ and the radial loading ranges from 335 to $411 \mathrm{kPa}$. The mass of these structures are calculated as according to [4].

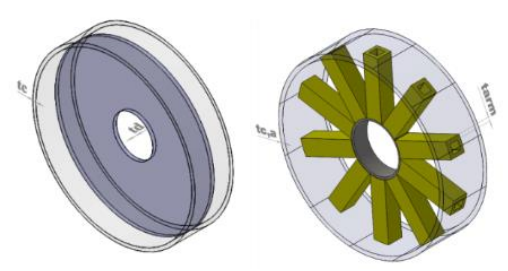

Figure 1: Rotor structures with dimensions as used in this study (a) with disc structure (b) with arm structure [4]

\subsection{Finite Element Approach}

For the finite element analyses, the model was constrained at the shaft and evaluated for Mode 1 deflection. Considering the variations of the flux density within the electromagnetic circuit, a maximum normal stress was located on the top of the structure while the minimum normal stress was placed at the bottom with the stress varying sinusoidally. An expansion load was applied to the rotor rim as explained. For the stator structures the same methodology was put into practice. A compression load acts on the inner bore of the stator cylinder.

The thicknesses of the cylinders, $t_{\mathrm{c}}$, the thicknesses of the discs in the case of the disc structures, $t_{\mathrm{d}}$, and the thicknesses of the arms in the case of arm structures, $t_{\text {arm }}$, were changed and the deflection was evaluated. For the armed structures, 10 ties of width, $w=0.35 \mathrm{~m}$, were employed. The material characteristics of these structures made up of steel are as follows: Young's modulus, $E=2.1 \times 10^{11} \mathrm{~Pa}$, Poisson's ratio, $v$ $=0.3$ and density, $\rho=7850 \mathrm{~kg} / \mathrm{m}^{3}$.

Once the deflection results were produced, these were then converted into stiffness by dividing by the peak applied force.

\subsection{Hybrid Approach}

The structural behaviour of the components forming the generator structure can be estimated with algebraic equations fitted to a combination of results obtained from dimensional studies [5] and a limited number of finite element analyses.

By way of illustration, this sub-section shows the hybrid method as applied to a disc sub-structure for a generator rotor structure. So as to find physically meaningful algebraic equations that accurately describe the behaviour of disc structures, analytical methods rooted in the principle of dimensional homogeneity were utilised. Assuming that the stiffness of the disc depends on the Young's modulus, E, thickness, $t_{\mathrm{d}}$, Poisson's ratio, $v$, and the difference between air-gap and shaft radii $(R-r)$, where $r$ is the radius of the shaft, then in terms of dimensions,

$$
\left[k_{\mathrm{s}, \mathrm{d}}\right]=\left[E^{a} t_{\mathrm{d}}^{b}(R-r)^{c}\right],
$$

where $a, b$ and $c$ are unknown. Using $[\mathrm{F}]$ and [L], to represent force and length dimensions, equation (3) can be reinterpreted as,

$$
\left[\mathrm{FL}^{-1}\right]=\left[\mathrm{F}^{a} \mathrm{~L}^{-2 a} \mathrm{~L}^{b} \mathrm{~L}^{c}\right]
$$


noting that Poisson's ratio is a dimensionless variable.

If powers are equated it is found that when $a=1$ and $-1=-2 a$ $+b+c$. In order to find $b$ and $c$, the thickness and the length are independently varied and the relationship to stiffness is observed. For this, the use of the FE data collected in Section 3.1 was required. Finally, the equation was balanced by introducing constants, leading to

$$
k_{\mathrm{s}, \mathrm{d}}=\frac{4160 t_{\mathrm{d}}{ }^{2} \mathrm{E}\left(1+v^{2}\right)}{\left(400 t_{\mathrm{d}}+R-r\right) \gamma}
$$

The implementation of another dimensionless variable, $\gamma$, which allows us to consider the deflection mode, was also introduced. $\gamma$ is equal to $\frac{\sigma_{\text {radial,max }}}{\sigma_{\text {radial,min }}}$ thus Mode 0 is by definition when $\gamma=1$.

Figure 2 gives a comparison between the data retrieved from equation (5) for Mode 0 and data from the FE simulation studies. As shown by the straight line of gradient 1, going through the origin, a good agreement found over the whole range. The data has an $R^{2}=0.9941$ for FE Disc Stiffness = Equation Disc Stiffness [3].

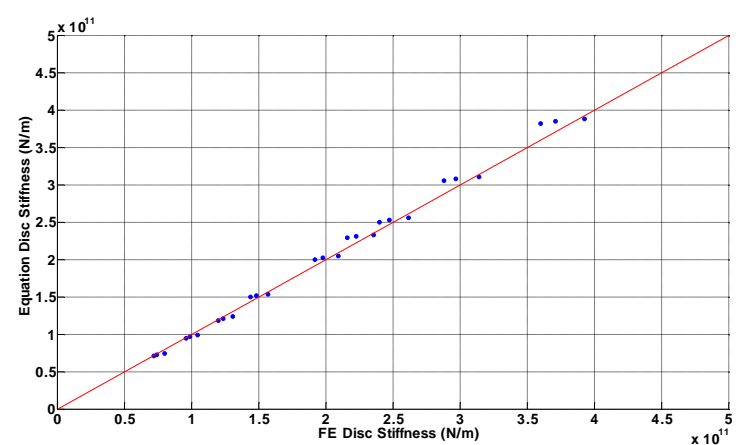

Figure 2: Comparison of a disc sub-structure stiffness evaluated by FE and using equation (5) [3]

With the cylinder and discs sub-structure stiffnesses calculated, the structure stiffness can be estimated by combining them in series, i.e.

$$
k_{\mathrm{s}}=\left(\frac{k_{\mathrm{eq}, \mathrm{d}} k_{\mathrm{c}}}{k_{\mathrm{eq}, \mathrm{d}}+k_{\mathrm{c}}}\right) \text {. }
$$

\subsection{Analytical Approach}

By using analytical methods, the stiffness of the substructures forming the rotor and the stator can also be found. In [3], Jaen-Sola developed a model capable of predicting the stiffness of a disc sub-structure by modifying Benham's model [6]. In [7], McDonald found the deflection of the arms and the deflection of the cylinder at the midpoint between arms using the forces acting on each sub-structure for Mode 0 deflection. The said forces were calculated by making use of compatibility equations.
In this paper, the authors derived an expression, which is able to estimate the required stiffness for arm sub-structures by looking at the physical features of the structure. The arms are generator sub-structures that connect the external cylinder to the main shaft in the case of the rotor. For the stator, these arms, also called ties, are attached to the turret. The aim of the arms is to stiffen the generator structure in order to withstand the large loads present during operation as well as during the transportation and installation stages.

Starting from Young's modulus one can start to relate the radial force applied to an arm and its deflection using stress and strain,

$$
E=\frac{\sigma_{\mathrm{arm}}}{\varepsilon_{\mathrm{arm}}}=\frac{\frac{F}{A_{\mathrm{arm}}}}{\frac{\delta}{l_{\mathrm{arm}}}}=\frac{F l_{\mathrm{arm}}}{A_{\mathrm{arm}} \delta},
$$

where $F$ is the force applied to the arm, $A_{\text {arm }}$ is the cross sectional area of the $\operatorname{arm}, \delta$ is the deflection in the longitudinal direction and $l_{\mathrm{arm}}$ is the length of the arm. Reordering the equation with $k=\frac{F}{\delta}$ and rearranging it is found that,

$$
k_{\text {arm }}=\frac{E A_{\text {arm }}}{l_{\text {arm }}} .
$$

If it is considered that the ties are hollow square structures of width, $w$, and thickness, $t_{\mathrm{arm}}$, then the cross sectional area is

$$
A_{\mathrm{arm}}=4 t_{\mathrm{arm}} w-4 t_{\mathrm{arm}}^{2}
$$

Substituting $A_{\text {arm }}$ into equation (8) the following expression for the arms structural stiffness can be found,

$$
k=\frac{4 E t_{\mathrm{arm}}\left(w-t_{\mathrm{arm}}\right)}{l_{\mathrm{arm}}} .
$$

A comparison between the results obtained from FE studies and those achieved with the equation is shown in Section 3.3.

\subsection{Optimisation}

Equations (2) and (3) indicate that for a given electromagnetic design (with a value of $k_{\mathrm{M}}$ ) there are many combinations of rotor and stator structure stiffness that meet (3). The optimisation process will minimise the sum of rotor and stator structural mass subject to meeting (3) and other constraints. In this case, this also includes a tangential deflection limit.

\section{Results}

In this section, results found by the three different types of approaches are given. The FE technique is shown first and the results are used as a benchmark to verify the other methods. An example of how the mass minimisation process should be carried out is explained in detailed. Then the data achieved with the hybrid and the analytical techniques are shown and validated. 


\subsection{Modelling Structural Stiffness using Finite Element Techniques}

\subsubsection{Disc Structure}

Figure 3 (a) and (b) are contour plots stiffness and mass for the disc rotor structure and a disc stator structure. The two axes show the two independent structural variables, and labelled contours show the stiffness and mass. These are interpolated based on $6 \times 6=36$ finite element simulations each. There are two further lines giving arbitrary tangential stiffness and radial stiffness 'limits' which correspond to deflection of $10 \%$ of the air-gap length.

Assuming a constant bearing stiffness $k_{\mathrm{b}}=3 \times 10^{9} \mathrm{~N} / \mathrm{m}$ and knowing that the structure needs to have a total stiffness $k_{\mathrm{s}}=1 \times 10^{9} \mathrm{~N} / \mathrm{m}$, a range of stator and rotor designs can be examined. In order to meet tangential stiffness requirements (by applying a torque of $2,250 \mathrm{kNm}$ to the structures), the thickness of the rotor disc must be at least equal to $40 \mathrm{~mm}$ whereas the thickness of the stator's discs must be over $20 \mathrm{~mm}$.

Considering all of these features, the minimum rotor structural mass can be estimated for the whole range of stiffnesses by looking at the plot presented in Figure 3(a), where the red line determines the minimum stiffness in the normal direction. Introducing $k_{\mathrm{s}, \mathrm{r}}$ and $k_{\mathrm{b}}$ into Equation (1), the equivalent rotor stiffness can be calculated. Equation (2) show that for every $k_{\text {eq, }}$ there is a minimum value of $k_{\mathrm{s}, \mathrm{s}}$ that is required. The numbered points in Figures 3 show a number of design models that meet the requirements.

The total structural mass of the machine is found by combining the rotor structure mass, $m_{\mathrm{s}, \mathrm{r}}$, and the stator structure mass, $m_{\mathrm{s}, \mathrm{s}}$ as can be seen in Figure 4. Model ' 1 ' is a stiff stator with a relatively compliant rotor; model ' 8 ' is the opposite with a more compliant stator structure and a stiffer rotor. The minimum mass for this generator structure, $m_{\mathrm{s}}$, is $18,500 \mathrm{~kg}$.

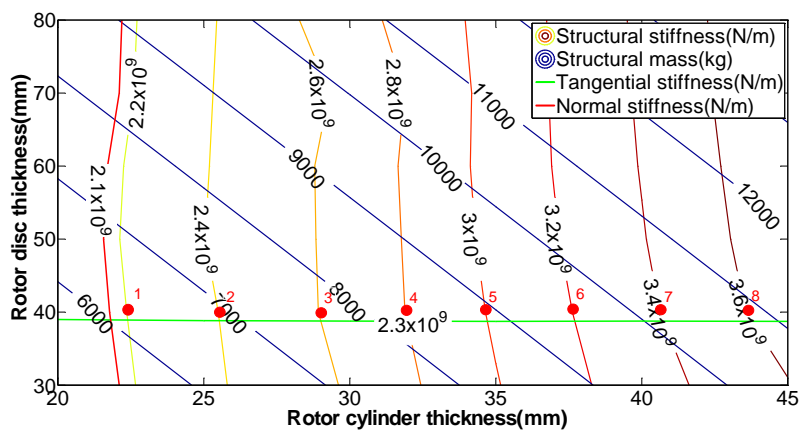

(a)

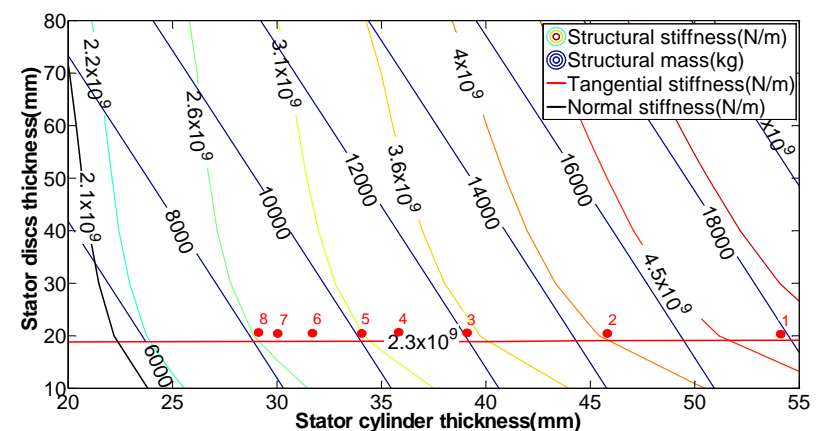

(b)

Figure 3: 2D optimisation for 3MW rotor and stator disc structures with structural stiffness criterion

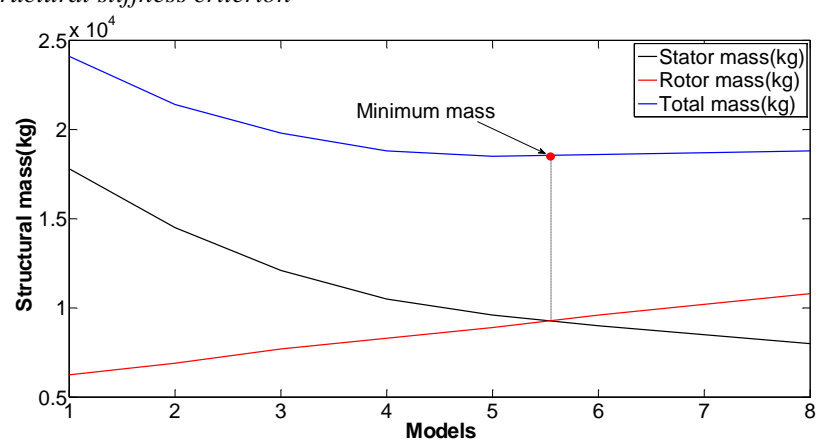

Figure 4: Mass optimisation result for disc structures

\subsubsection{Armed Structure}

Following the same methodology the minimum mass for the armed structures case can be found. See Figure 5. For the same inputs and constraints, the minimum generator mass was $35,500 \mathrm{~kg}$ as seen in Figure 6.

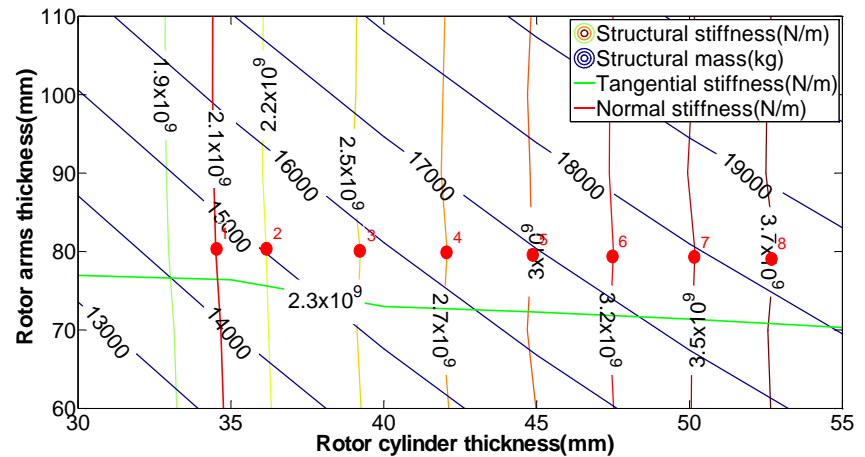

(a)

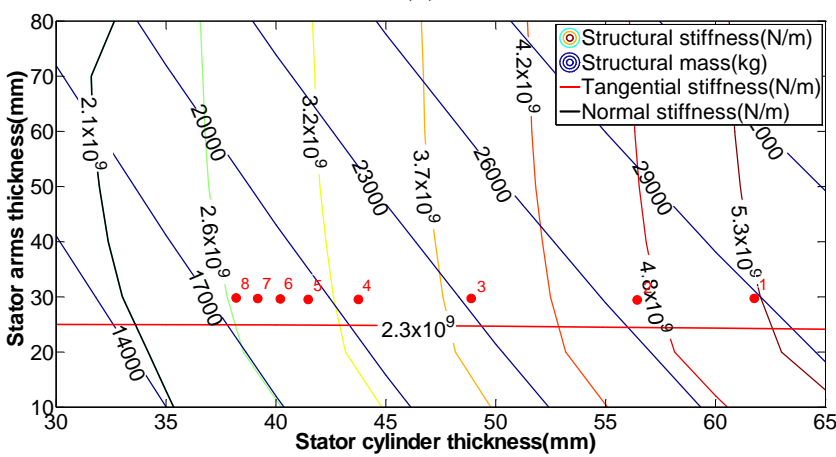

(b)

Figure 5: 2D optimization for 3MW rotor and stator armed structures with structural stiffness criterion 


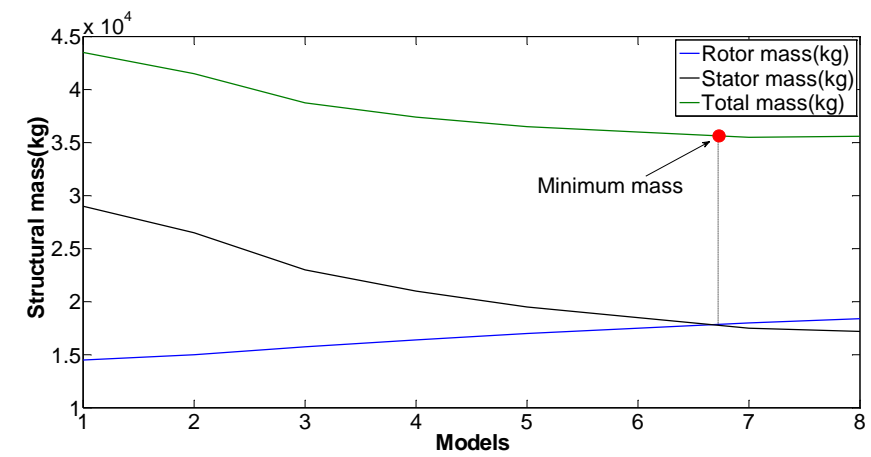

Figure 6: Mass optimisation result for armed structures

\subsection{Modelling Structural Stiffness with a Hybrid Approach}

The hybrid approach was presented by the authors in [3], where the stiffness of the components forming a disc rotor structure and the stiffness of the rotor itself were estimated obtaining satisfactory results. In this investigation, the stiffness of the disc stator structure and its components and the stiffness of the elements of an armed structure and the armed structure itself have been calculated.

\subsubsection{Stator with Discs Stiffness Model}

The stator's structural stiffness was predicted by putting together the cylinder, $k_{\mathrm{c}, \mathrm{s}}$, and the discs structures in series. Because the two discs are in parallel, they are added together to give an equivalent stiffness, $k_{\mathrm{eq}, \mathrm{d}}=k_{\mathrm{d} 1}+k_{\mathrm{d} 2}$.

The same approach that was used to find out the equation of the rotor in [3] was utilised in the case of the stator. As the stator discs are constrained in the same way as it was done with the rotor disc, Equation (5) was considered valid. However, a new formula for the stiffness of the cylinder was needed.

\subsubsection{Stator Cylinder Sub-Structure Model}

As illustarted in Section 2.3, a dimensional analysis of the component in question was made. In this particular case it was assumed that the stiffness of the cylinder depends on the Young's Modulus, $E$, thickness, $t_{\mathrm{c}, \mathrm{s}}$, length, $l_{\mathrm{c}, \mathrm{s}}$, radius, $R_{\mathrm{c}, \mathrm{s}}$ and Poisson's ratio, $v$. As expected, the study could not predict all the powers of the variables present in the equation, therefore they had to be determined by analysing the variation of each parameter with stiffness. A constant had to be introduced to balance the equation.

$$
k_{\mathrm{c}}=3.82 \times 10^{10}+\left[\frac{442.14 E t_{\mathrm{c}, \mathrm{s}}^{2} l_{\mathrm{c}, \mathrm{s}}\left(1+v^{2}\right)}{R_{\mathrm{c}, \mathrm{s}}^{2} \gamma}\right]
$$

Comparing the equation retrieved data and the FE studies results for Mode 0, it can be observed that a reasonable level of accuracy was obtained, as a straight line of gradient 1 , passing through the origin, fits the data with an $R^{2}$ equal to 0.9225 .

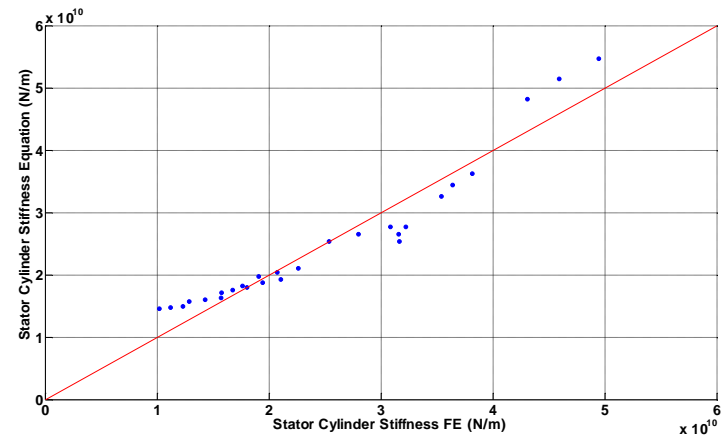

Figure 7: Stator cylinder Equation vs. FE

Although an overall good match between the results from (5) and (6) and the FE studies for the whole structure was found, there were some weaknesses. It was seen that as the radius increases, for considerably large disc thicknesses (over $150 \mathrm{~mm}$ ) and short cylinder lengths, the model underestimates the stator stiffness. This is because the equation predicts a lower contribution of the discs to the overall stiffness. This implies that the equations for the hybrid method should only be used in limited ranges of dimensions.

\subsubsection{Cylinder Sub-Structure Model for Armed Rotor}

In this case, it was assumed that $k_{\mathrm{c}, \mathrm{a}}=f\left(E, t_{\mathrm{c}, \mathrm{a}}, l_{\mathrm{c}, \mathrm{a}}, R_{\mathrm{c}, \mathrm{a}}, v\right)$. Once the dimensional analysis and sensitivity analysis was completed and the retrieved data were analysed, Equation (12) was found.

$$
k_{\mathrm{c}, \mathrm{a}}=1 \times 10^{10}+\left[\frac{86.35 E t_{\mathrm{c}, \mathrm{a}}^{2}\left(1+l_{\mathrm{c}, \mathrm{a}}^{2}\right)\left(1+v^{2}\right)}{R_{\mathrm{c}, \mathrm{a}}^{2} l_{\mathrm{c}, \mathrm{a}} \gamma}\right]
$$

As seen in Figure 8, a fair precision was achieved. Nevertheless, higher volatility can be appreciated for models corresponding to cylinders with very large thicknesses $(150 \mathrm{~mm})$. The equality is considered valid for a range between 5 and 7 metres diameter. The results have a $R^{2}$ value of 0.9454 regarding a straight line of gradient 1 passing through the origin.

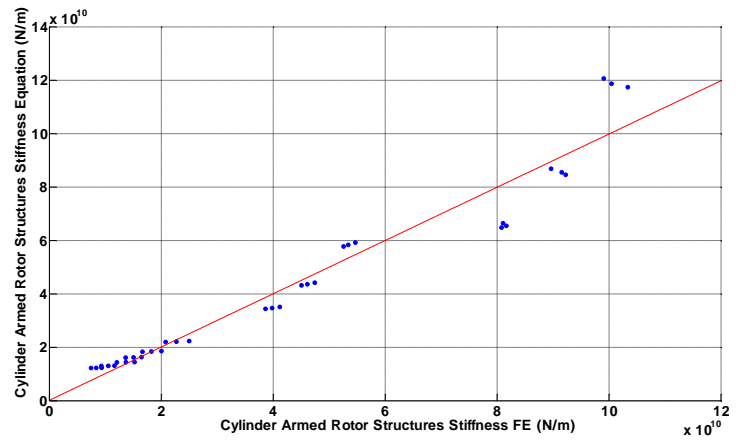

Figure 8: Armed rotor cylinder Equation vs. FE 


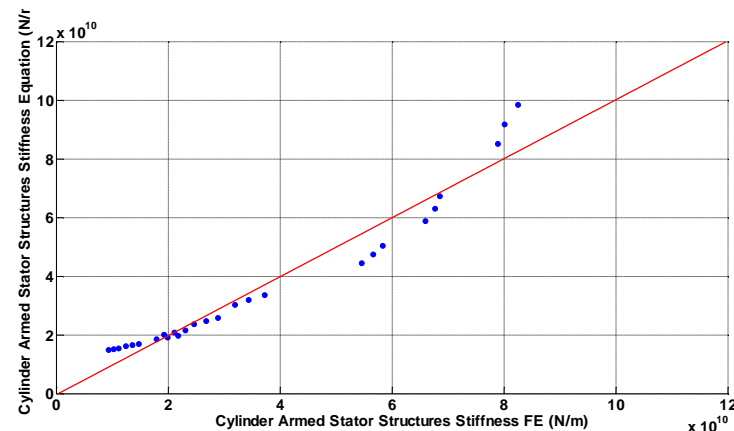

Figure 9: Armed stator cylinder Equation vs. FE

\subsubsection{Cylinder Sub-Structure Model for Armed Stator}

Using the same arguments as for Section 3.2.3, the final equation to find the cylinder stiffness is as follows,

$$
k_{\mathrm{as}, \mathrm{c}}=1.19 \times 10^{10}+\left[\frac{128.44 E t_{\mathrm{as}, \mathrm{c}}^{2} l_{\mathrm{as}, \mathrm{c}}\left(1+v^{2}\right)}{R_{\mathrm{as}, \mathrm{c}}^{2}}\right]
$$

A comparison between the results obtained from the equation for the cylinder under Mode 0 deflection and the data acquired from the FE simulation studies was made. As it can be seen in Figure 9, a good agreement was achieved again as the data has an $R^{2}=0.9455$.

\subsubsection{Armed Rotor and Stator Stiffness Models}

Having the equivalent stiffness of the arms, $k_{\mathrm{a} \text {,eq }}$, and the stiffness of the cylinder, $k_{\mathrm{c}, \mathrm{a}}$, the stiffness of the rotor can be calculated. However, the use of such method does not seem to be effective as the data obtained from combining substructures did not show a good agreement with the FE results.

The stator's cylinder stiffness was also estimated using the method described in Section 3.2.4 whereas the equation acquired for the rotor arms in Section 3.3 was recognized as valid since the same constraints and forces were applied. In spite of the fact that both equations, for cylinder and arm, produced very valuable data, it was not possible to achieve good results when combining them together into the stator's equation.

\subsection{Modelling Structural Stiffness through Analytical Methods}

Figure 10 shows the comparison between the data obtained from FE analyses of arms and the values found with equation (10).

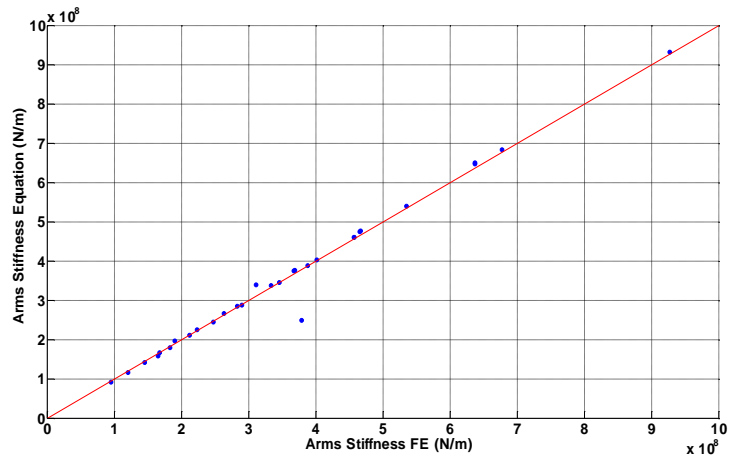

Figure 10: Comparison of stiffness estimated with analytical model and FE

\section{Discussion \& Conclusions}

Mass optimisation of the supporting structure of radial-flux PM generators for direct-drive wind turbines has been carried out making use of stiffness criteria. Data utilised in this study were obtained from different models: analytical, FE and hybrid, which combines results from FE studies with dimensional studies data. As demonstrated, analytical methods perform well when looking at Mode 0 deflection for simple sub-structures whereas either FE or hybrid must be used if Mode 1 or above are to be analysed. Nevertheless, it is worth highlighting that the hybrid approach underestimates the required stiffness for full discontinuous assemblies, such as armed structures, thus it is not suitable for analysing this type of configurations. It was observed that the cylinder stiffness was the dominant parameter, whereas the equivalent stiffness of the arms did not have enough weight leading the equation to heavily underestimate the stiffness.

The use of the stiffness criterion either on the rotor or stator design separately does not guarantee the integrity of the supporting structure, but considering them together can lead to mass optimisation which meets all the stiffness requirements. According to the mass optimisation study, disc structures are lighter than armed structures as the study revealed a difference of $18,000 \mathrm{~kg}$ between the two. This is because armed structures do not perform well under torque loads unless the thickness of the hollow arms is significantly increased with a consequent rise in mass. The design of the machine must be done considering all the components forming the generator at the same time.

\section{References}

[1] J. N. Stander, G. Venter, and M. J. Kamper, "Review of direct-drive radial flux wind turbine generator mechanical design," Wind Energy, 2012;15(3):459-472, doi:10.1002/we.484.

[2] M. Mueller, H. Polinder, and A. S. McDonald, "Structural mass in direct-drive permanent magnet 
electrical generators," IET Renew. Power Gener., vol. 2, no. 1, pp. 3-15, Mar. 2008.

[3] P. Jaen-Sola and A. S. McDonald, "Structural Analysis and Characterization of Radial Flux PM Generators for Direct-Drive Wind Turbines," 3rd International Renew. Power Gener. Conf. (IET RPG), Naples, Italy, 2014.

[4] A. S. McDonald and P. Jaen-Sola, "A Stiffness Approach for Coupling Structural and Magnetic Models for the Design and Optimization of Radial Flux PM Generators for Direct-Drive Wind Turbines," IET Renew. Power Gener. Journal, Submitted Jan. 2015.

[5] R. Pankhurst, "Dimensional Analysis and Scale Factors." London: The Institute of Physics and The Physical Society, 1964.

[6] P. Benham, R. Crawford and C. Armstrong, "Mechanics of Engineering Materials", Prentice Hall, Second Edition (1996).

[7] A. S. McDonald, "Structural analysis of low speed, high torque electrical generators for direct drive renewable energy converters," $\mathrm{PhD}$ thesis, University of Edinburgh, Edinburgh, 2008. 